\title{
Continuous-terahertz-wave molecular imaging system for biomedical applications
}

Rui Zhang

Liangliang Zhang

Tong $\mathrm{Wu}$

Ruixue Wang

Shasha Zuo

Dong $\mathrm{Wu}$

Cunlin Zhang

Jue Zhang

Jing Fang 


\title{
Continuous-terahertz-wave molecular imaging system for biomedical applications
}

\author{
Rui Zhang, ${ }^{\text {a }}$ Liangliang Zhang, ${ }^{\mathrm{b}, *}$ Tong Wu, ${ }^{\mathrm{b}}$ Ruixue Wang, ${ }^{\mathrm{c}}$ Shasha Zuo, ${ }^{\mathrm{c}}$ Dong Wu, ${ }^{\mathrm{c}}$ Cunlin Zhang, ${ }^{\mathrm{b}}$ \\ Jue Zhang, ${ }^{a, c, *}$ and Jing Fang ${ }^{a, c}$

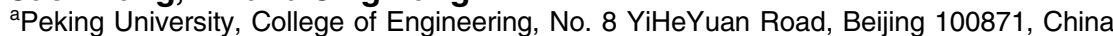 \\ ${ }^{b}$ Capital Normal University, Department of Physics, No. 105 XiSanHuan BeiLu, Beijing 100048, China \\ 'Peking University, Academy for Advanced Interdisciplinary Studies, No. 8 YiHeYuan Road, Beijing 100871, China
}

\begin{abstract}
Molecular imaging techniques are becoming increasingly important in biomedical research and potentially in clinical practice. We present a continuous-terahertz (THz)-wave molecular imaging system for biomedical applications, in which an infrared (IR) laser is integrated into a 0.2-THz reflection-mode continuous-THz-wave imaging system to induce surface plasmon polaritons on the nanoparticles and further improve the intensity of the reflected signal from the water around the nanoparticles. A strong and rapid increment of the reflected $\mathrm{THz}$ signal in the nanoparticle solution upon the IR laser irradiation is demonstrated, using either gold or silver nanoparticles. This low-cost, simple, and stable continuous-THz-wave molecular imaging system is suitable for miniaturization and practical imaging applications; in particular, it shows great promise for cancer diagnosis and nanoparticle drug-delivery monitoring. ๑ 2016 Society of Photo-Optical Instrumentation Engineers (SPIE) [DOI: 10.1117/1.JBO.21.7 .076006]
\end{abstract}

Keywords: continuous-terahertz-wave; nanoparticle; molecular imaging.

Paper 160067R received Feb. 1, 2016; accepted for publication May 23, 2016; published online Jul. 12, 2016.

\section{Introduction}

Molecular imaging techniques have been widely employed to image particular targets or pathways in various biomedical imaging modalities, ${ }^{1}$ such as magnetic resonance imaging, computed tomography, single-photon emission computed tomography, positron emission tomography, and fluorescence imaging. ${ }^{2-5}$ To achieve molecular imaging, there are three essential factors: a high-specificity molecular probe, an efficient amplification strategy, and a high-sensitivity imaging system. ${ }^{6}$

Recently, terahertz ( $\mathrm{THz}$ ) imaging has been widely applied to distinguish lesions from normal tissues ${ }^{7-10}$ because $\mathrm{THz}$ radiation is nonionizing and sensitive to water and to the motion of biological macromolecules. ${ }^{11}$ Furthermore, to improve image contrast between diseased and normal tissues, nanoparticleenhanced $\mathrm{THz}$ molecular imaging techniques have been proposed $^{12-14}$ and applied to cancer diagnosis ${ }^{15}$ and drugdelivery detection. ${ }^{16}$ Various nanoparticles, such as gold nanoparticles (AuNPs) ${ }^{12,13}$ or superparamagnetic iron oxide nanoparticles, ${ }^{14}$ were adopted as imaging probes. The main reason for signal amplification is that the power absorption and refractive index of water are temperature dependent in the $\mathrm{THz}$ region. ${ }^{17}$ Under infrared (IR) laser irradiation, surface plasmon polaritons are produced on the nanoparticles; this increases the temperature of water in the tissue containing nanoparticle contrast. By using a reflection-mode $\mathrm{THz}$ time-domain spectroscopic (THz-TDS) system, a highly sensitive THz reflection signal was observed in tissues incorporated with nanoparticles under the IR light. Moreover, a strong image contrast was obtained between tissues with and without nanoparticle contrast in the differential $\mathrm{THz}$ image. ${ }^{18}$

*Address all correspondence to: Liangliang Zhang, E-mail: zhlliang@126.com; Jue Zhang, E-mail: zhangjue@pku.edu.cn
Nonetheless, THz-TDS systems were adopted in former THz molecular imaging techniques. ${ }^{12-16,18}$ The system compositions for generation and coherent detection of $\mathrm{THz}$ pulses are rather complex for practical applications, especially for monitoring surgical processes in the operating room. In addition, the $\mathrm{THz}$ pulses can be strongly absorbed by water vapor in the air for the high power absorption of water in the adopted $\mathrm{THz}$ region $(0.1$ to $3 \mathrm{THz}) .{ }^{17}$ In contrast, the continuous-THz-wave imaging system, which has also been widely employed for biomedical imaging of various tissues, is simple, low cost, and stable. ${ }^{19-22}$ Also, the imaging speed of the continuous-THz-wave imaging system is always faster than the pulsed imaging system without the need for coherent detection of $\mathrm{THz}$ pulses. In addition, the absorption of water vapor at low $\mathrm{THz}$ frequencies is small ${ }^{17}$ and thus the continuous-THz-wave imaging system could be more feasible for practical applications.

In this study, a continuous-THz-wave molecular imaging system was proposed, in which an IR laser was incorporated into the $0.2-\mathrm{THz}$ reflection-mode continuous-THz-wave imaging system to induce surface plasmon polaritons on the nanoparticles and further enhance the reflected signal intensity of water around the nanoparticles. To test the feasibility of this system, two nanoparticle contrasts that are widely used in other molecular imaging modalities, namely, AuNPs and silver nanoparticles (AgNPs), ${ }^{23}$ were employed to investigate the changes in signal response under the IR modulation.

\section{Experimental Method and Materials}

The AuNPs and AgNPs used in this study were synthesized by a previously reported cold plasma approach with an average diameter of $18.3 \pm 7.4$ and $12.1 \pm 2.3 \mathrm{~nm}$, respectively. ${ }^{24,25}$

$1083-3668 / 2016 / \$ 25.00$ @ 2016 SPIE 
The nanoparticle water solutions were adopted to simulate the $\mathrm{THz}$ response from cells after endocytosis of nanoparticle contrast because tissues, especially cancerous tissues, contain a large amount of interstitial water. ${ }^{18}$

The reflection-mode continuous-THz-wave molecular imaging system is shown in Fig. 1. The THz radiation generated by the 0.2-THz Gunn oscillator (Radiometer Physics) was focused on the sample by a polyethylene lens. The diameter of the $\mathrm{THz}$ wave beam on the sample surface was about $4 \mathrm{~mm}$. The reflected $\mathrm{THz}$ wave was refocused by a parabolic mirror. The refocused $\mathrm{THz}$ wave beam was detected by the pyroelectric detector (Microtech SN:171344). The distance between the parabolic mirror and the pyroelectric detector and that between the parabolic mirror and sample were accurately adjusted to satisfy the imaging equation. The output signal from the pyroelectric detector was fed into a lock-in amplifier referenced to the $15-\mathrm{Hz}$ modulation frequency. In addition, a compact femtosecond fiber laser (IMRA Feomtolite FX-100) with a repetition rate of $75 \mathrm{MHz}$, central wavelength of $800 \mathrm{~nm}$, and pulse duration of $100 \mathrm{fs}$ was adopted as a source of IR irradiation to induce surface plasma resonance on the nanoparticles in this system. The IR laser beam was adjusted to overlap with the $\mathrm{THz}$ beam at the same spot on the sample surface with a diameter of $3 \mathrm{~mm}$. To test the feasibility and universality of this continuous-THz-wave molecular imaging modality, the reflected $\mathrm{THz}$ signals from the AuNP and AgNP solutions were recorded for different nanoparticle solution densities and under different IR laser intensities. The minimum solution density for AuNPs or AgNPs was determined to induce a reflection change of about $1 \%$ in $10 \mathrm{~s}$ under the IR laser illumination with an intensity of $1.0 \mathrm{~W} / \mathrm{cm}^{2}$.

\section{Results and Discussion}

The IR laser intensity dependence of the reflected $\mathrm{THz}$ signal from the AuNP solution with a concentration of $50 \mu \mathrm{g} / \mathrm{mL}$ is shown in Figs. 2(a) and 2(b). The amplitude of the reflected $\mathrm{THz}$ signal from the AuNP solution $(50 \mu \mathrm{g} / \mathrm{mL})$ increases dramatically upon switching on the IR laser (at $0 \mathrm{~s}$ ), whereas there is almost no change in its absence. In addition, the reflection change increases as the intensity of the IR laser increases. In our experiment, the relative reflection amplitude change was about $10 \%$ for the $50 \mu \mathrm{g} / \mathrm{mL}$ AuNP solution after $120 \mathrm{~s}$ of exposure to the IR laser with the intensity of $1.0 \mathrm{~W} / \mathrm{cm}^{2}$. The AuNP solution density dependence of the reflected $\mathrm{THz}$ signal under the IR laser with the intensity of $1.0 \mathrm{~W} / \mathrm{cm}^{2}$ is shown in Figs. 2(c) and 2(d). A clear reflection change is observed even for a low concentration of $12.5 \mu \mathrm{g} / \mathrm{mL}$. However, there is almost no amplitude change in the THz signal reflected from water without AuNPs under the IR laser irradiation. In addition, the relative reflection amplitude change increases with an increase in the AuNP solution density.

To further demonstrate the universality of this continuousTHz-wave molecular imaging system, AgNPs were also tested as the contrast agent. Due to the different properties of the surface plasmon resonance effect between AgNPs and AuNPs, the minimum AgNPs solution density was chosen as $125 \mu \mathrm{g} / \mathrm{mL}$ to induce a reflection change of about $1 \%$ in $10 \mathrm{~s}$ under the IR laser illumination with an intensity of $1.0 \mathrm{~W} / \mathrm{cm}^{2}$. The IR laser intensity and solution density dependence of the reflected $\mathrm{THz}$ signal from the AgNP solution are shown in Fig. 3. Similar to the case of the AuNPs, the amplitude of reflected $\mathrm{THz}$ signal from the AgNP solution increases dramatically upon switching on the IR laser (at $0 \mathrm{~s}$ ), whereas there is almost no change in the absence of the IR laser or from water without AgNPs. Furthermore, the reflection change increases with the intensity of the IR laser and with the AgNP solution density. In our experiment, the relative reflection amplitude change was about $7 \%$ for the $500 \mu \mathrm{g} / \mathrm{mL} \mathrm{AgNP}$ solution after $120 \mathrm{~s}$ of exposure to the IR laser with an intensity of $1.0 \mathrm{~W} / \mathrm{cm}^{2}$.

The nanoparticles themselves have no influence on the reflected $\mathrm{THz}$ signal, and there is no interaction between the nanoparticles and the $\mathrm{THz}$ wave. This is because of negligible reflection or absorption of $\mathrm{THz}$ waves by the nanoparticles, considering that the $\mathrm{THz}$ wavelength is three to four orders of magnitude larger than the nanoparticle dimensions. ${ }^{18}$ Moreover, the IR laser itself also has no effect on the reflected $\mathrm{THz}$ signal. Nevertheless, the IR irradiation of the nanoparticles leads to an increase in the water temperature due to the surface plasma resonance effect. ${ }^{18}$ Higher water temperature was achieved with higher IR laser intensity and nanoparticle solution density, which resulted in a stronger reflected $\mathrm{THz}$ signal for the higher refractive index and power absorption of water at higher temperatures. $^{17}$

The magnified curves for the reflection changes as a function of time in the range from -5 to $+30 \mathrm{~s}$ for AuNP $(50 \mu \mathrm{g} / \mathrm{mL})$ and AgNP $(500 \mu \mathrm{g} / \mathrm{mL})$ solutions under the irradiation of the IR laser with an intensity of $1.0 \mathrm{~W} / \mathrm{cm}^{2}$ are shown in Fig. 4. During the first $10 \mathrm{~s}$ after the IR laser irradiation begins, the amplitude of the reflected THz signal from the AgNP solution has a quicker and stronger enhancement than that from the

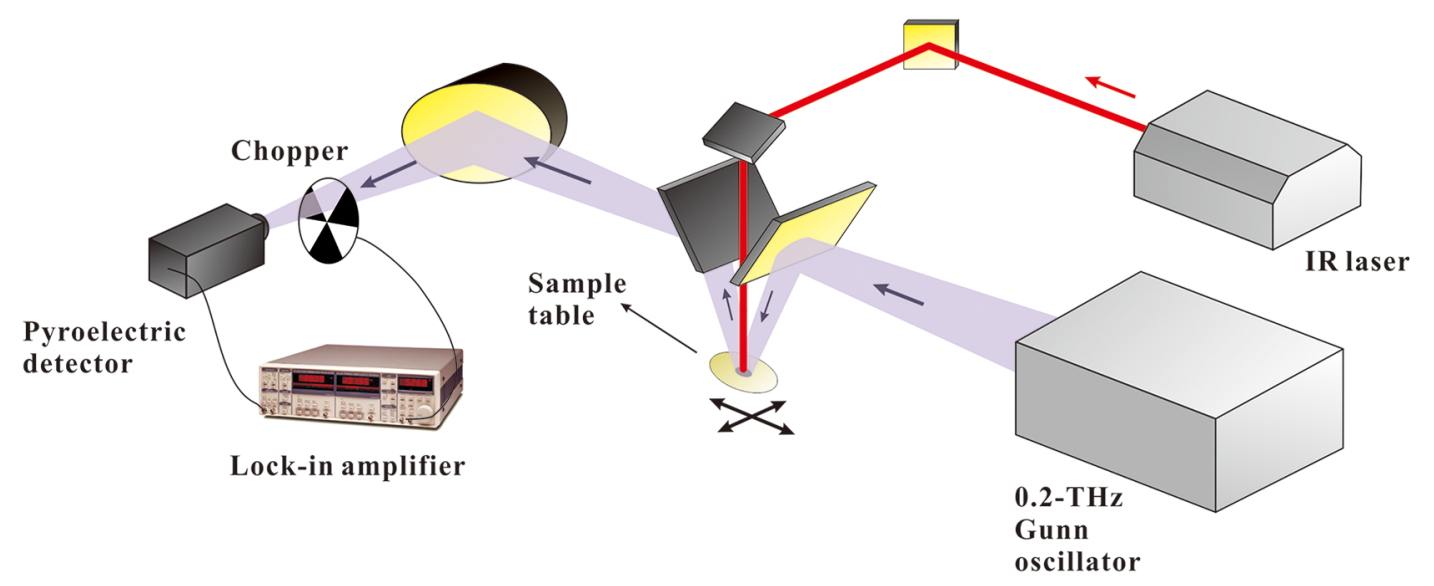

Fig. 1 The reflection-mode continuous-THz-wave molecular imaging system (0.2 THz). 

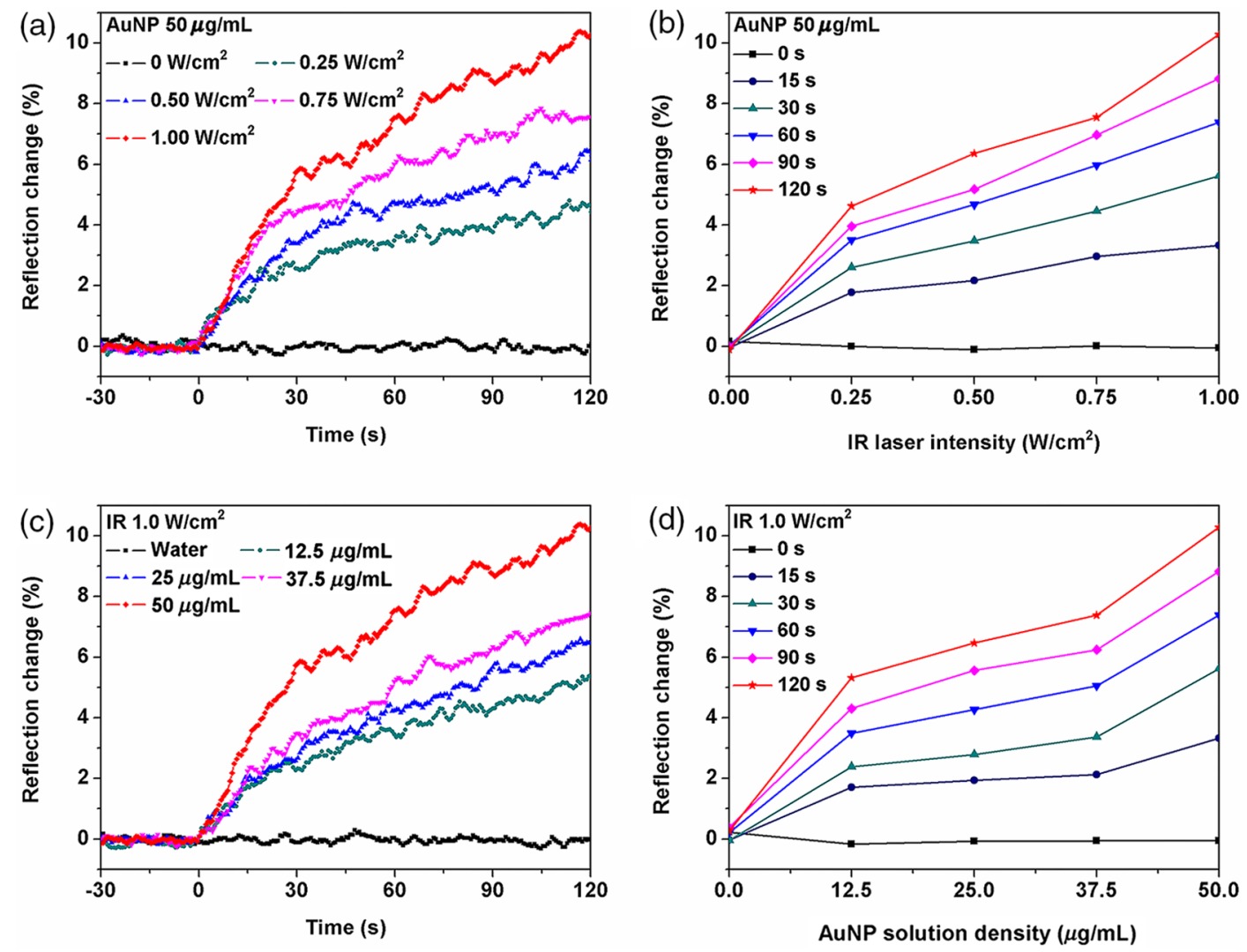

Fig. 2 ( $a$ and b) IR laser intensity dependence of the reflected THz signal from an AuNP solution with a concentration of $50 \mu \mathrm{g} / \mathrm{mL}$. (a) Time-varying reflection changes for varying IR laser intensities and (b) reflection changes as a function of IR laser intensity at different time instants. (c and d) AuNP solution density dependence of the reflected $\mathrm{THz}$ signal under IR laser illumination with an intensity of $1.0 \mathrm{~W} / \mathrm{cm}^{2}$. (c) Time-varying reflection changes for various AuNP solution concentrations and (d) reflection changes with AuNP solution density at various time instants.

AuNP solution. In the 10 to $30 \mathrm{~s}$ range, the reflection change from AuNP solution continues to increase, whereas the enhancement of the AgNP solution is close to saturation. At $30 \mathrm{~s}$, the reflection change from the AuNP solution is higher than that from the AgNP solution. These results demonstrate that the adopted AuNPs and AgNPs in our experiment have different time-varying heating effects induced by surface plasma resonance. The reflection change is also highly dependent on the properties of the applied nanoparticles, except for the IR laser intensity and the nanoparticle solution density.

For pixel-by-pixel imaging applications using the raster scan technique, the reflection change within the first few seconds after the IR laser irradiation is crucial. For both AuNP and AgNP solutions adopted in this experiment, the reflection enhancement occurred instantly upon the IR laser illumination, which makes it possible to achieve pixel-by-pixel imaging at high speed. In addition, the instant reflection change within a few seconds after the IR laser irradiation is highly dependent on the property of the nanoparticle contrast, the IR laser intensity, and the nanoparticle solution density. Stronger and more rapid reflection enhancement could be further achieved by employing nanoparticles with larger and faster heating effects in a future study.

These preliminary results indicate that the proposed continuous-THz-wave molecular imaging system shows potential for cancer diagnosis and nanoparticle drug-delivery monitoring. Moreover, the contour of cancerous tumors could be recognized in the images of differential and relative reflection change when surface-modified nanoparticles are specifically targeted to the tumor cells. ${ }^{18}$ An in vivo $\mathrm{THz}$ imaging study with high-specificity nanoparticles based on the proposed continuous-THzwave molecular imaging system will be implemented in the near future. In addition, photothermal therapy has also been widely applied to treat cancer, ${ }^{26}$ and the proposed imaging system can be applied for synchronously monitoring the heating effect in cancer tissues during the treatment, which will form the basis of another future study. Furthermore, this proposed $\mathrm{THz}$ imaging modality can be utilized in combination with other imaging modalities to achieve multimodality cancer imaging. ${ }^{1-6}$

In comparison with previous pulsed $\mathrm{THz}$ molecular imaging studies, ${ }^{12-16,18}$ the proposed continuous-THz-wave molecular imaging system possesses the advantages of low cost, high stability, simplicity of use, and suitability for miniaturization in portable medical systems. Meanwhile, the imaging speed is faster than previous pulsed imaging systems without the need for coherent detection of $\mathrm{THz}$ pulses. In addition, the absorption of $\mathrm{THz}$ wave by water vapor at low $\mathrm{THz}$ frequencies is small, ${ }^{17}$ thus, this system is more feasible for practical applications. Moreover, this continuous-THz-wave molecular imaging system can be further optimized: other high-quality, high-power continuous-THz-wave sources such as the backward-wave oscillator can be integrated into this system to further improve the signal-to-noise ratio and image resolution, and the Golay cell 

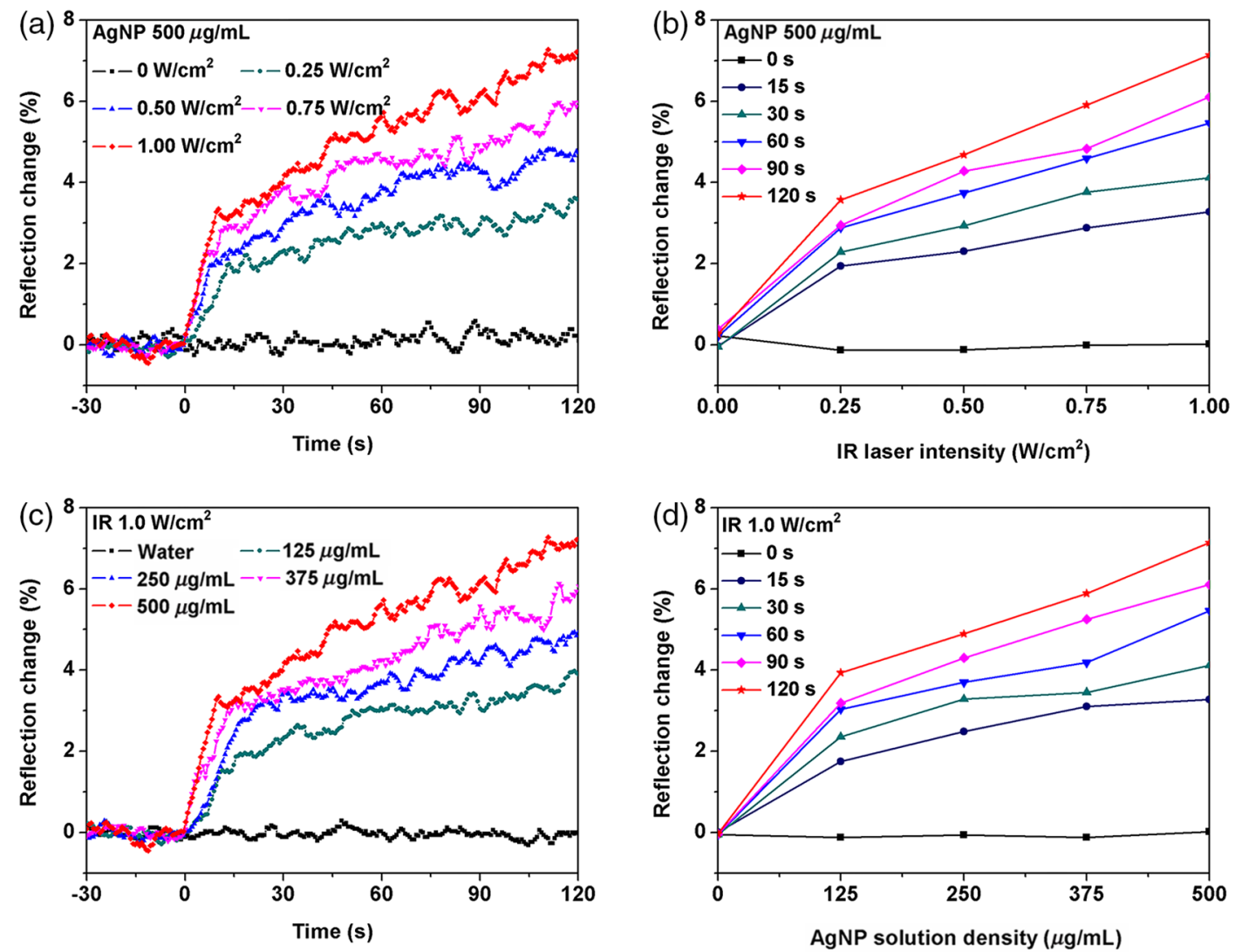

Fig. 3 ( $a$ and b) IR laser intensity dependence of the reflected THz signal from an AgNP solution with a concentration of $500 \mu \mathrm{g} / \mathrm{mL}$. (a) Time-varying reflection changes for varying IR laser intensities and (b) reflection changes as a function of IR laser intensity at different time instants. (c and d) AgNP solution density dependence of the reflected $\mathrm{THz}$ signal under IR laser illumination with an intensity of $1.0 \mathrm{~W} / \mathrm{cm}^{2}$. (c) Time-varying reflection changes for various AgNP solution concentrations and (d) reflection changes with AgNP solution density at various time instants.

can be introduced to replace the pyroelectric detector to further enhance the measurement sensitivity. Meanwhile, other kinds of high-quality continuous IR lasers can also be applied to induce efficient surface plasma resonance effect on the nanoparticles for in vivo applications.

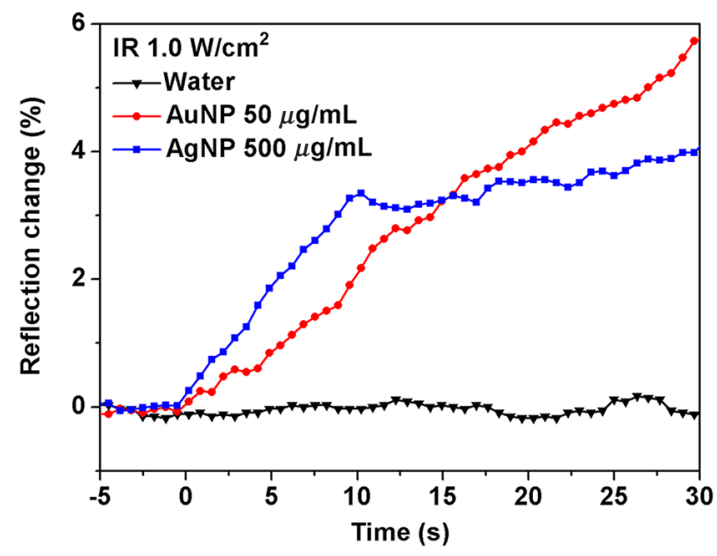

Fig. 4 Enlarged view of the time-varying reflection changes (in the range from -5 to $+30 \mathrm{~s}$ ) for the AuNP solution with a concentration of $50 \mu \mathrm{g} / \mathrm{mL}$ and the AgNP solution with a concentration of $500 \mu \mathrm{g} / \mathrm{mL}$ under IR laser illumination with an intensity of $1.0 \mathrm{~W} / \mathrm{cm}^{2}$.

\section{Conclusion}

In conclusion, we proposed a continuous- $\mathrm{THz}$-wave molecular imaging system. The system offers the advantages of low cost and simplicity of operation, and it is suitable for miniaturization and practical applications. Our preliminary results demonstrated that highly sensitive signal enhancement was obtained from water with AuNPs or AgNPs under the IR laser irradiation, whereas there was almost no change from water without AuNPs or AgNPs. Furthermore, the reflection change increases when the intensity of the IR laser and/or the nanoparticle solution density increase. We believe that our proposed continuous-THz-wave molecular imaging system will be very useful for cancer diagnosis and nanoparticle drug-delivery monitoring.

\section{Acknowledgments}

The authors acknowledge support from the National Natural Science Foundation of China under Grant No. 11374007 and the Foundation for the Author of National Excellent Doctoral Dissertation of PR China under Grant No. 201237. This work was funded by the National Keystone Basic Research Program (973 Program) under Grant No. 2014CB339806-1. This study was also supported by the Hong Kong Scholars Program through Project G-YZ53. 


\section{References}

1. M. Thakur and B. C. Lentle, "Report of a summit on molecular imaging," Radiology 236(3), 753-755 (2005).

2. D.-E. Lee et al., "Multifunctional nanoparticles for multimodal imaging and theragnosis," Chem. Soc. Rev. 41(7), 2656-2672 (2012).

3. J. Garcia, T. Tang, and A. Y. Louie, "Nanoparticle-based multimodal PET/MRI probes," Nanomedicine 10(8), 1343-1359 (2015).

4. Y. Wu et al., "Lanthanide-based nanocrystals as dual-modal probes for SPECT and X-ray CT imaging," Biomaterials 35(16), 4699-4705 (2014).

5. J. Zhang et al., "In vivo tumor-targeted dual-modal fluorescence/CT imaging using a nanoprobe co-loaded with an aggregation-induced emission dye and gold nanoparticles," Biomaterials 42, 103-111 (2015).

6. R. Weissleder, "Molecular imaging: exploring the next frontier," Radiology 212(3), 609-614 (1999).

7. P. Tewari et al., "In vivo terahertz imaging of rat skin burns," J. Biomed. Opt. 17(4), 040503 (2012).

8. M. H. Arbab et al., "Terahertz spectroscopy for the assessment of burn injuries in vivo," J. Biomed. Opt. 18(7), 077004 (2013).

9. K. Meng et al., "Terahertz pulsed spectroscopy of paraffin-embedded brain glioma," J. Biomed. Opt. 19(7), 077001 (2014).

10. Y. B. Ji et al., "Feasibility of terahertz reflectometry for discrimination of human early gastric cancers," Biomed. Opt. Express 6(4), 1398-1406 (2015).

11. S. T. Fan et al., "The growth of biomedical terahertz research," J. Phys. D: Appl. Phys. 47(37), 374009 (2014).

12. S. J. Oh et al., "Nanoparticle-enabled terahertz imaging for cancer diagnosis," Opt. Express 17(5), 3469-3475 (2009).

13. S. J. Oh et al., "Molecular imaging with terahertz waves," Opt. Express 19(5), 4009-4016 (2011).

14. J. Y. Park et al., "In vivo dual-modality terahertz/magnetic resonance imaging using superparamagnetic iron oxide nanoparticles as a dual contrast agent," IEEE Trans. Terahertz Sci. Technol. 2(1), 93-98 (2012).
15. S. J. Oh et al., "Cancer diagnosis by terahertz molecular imaging technique," J. Infrared Millimeter Terahertz Waves 33(1), 74-81 (2012).

16. J.-H. Son et al., "Imaging of nanoparticle delivery using terahertz waves," in Intracellular Delivery: Fundamentals and Applications, Fundamental Biomedical Technologies, A. Prokop, Ed., Vol. 5, pp. 701-711, Springer, Berlin (2011).

17. C. Ronne et al., "Investigation of temperature dependence of dielectric relaxation in liquid water by $\mathrm{THz}$ reflection spectroscopy and molecular dynamics simulation," J. Chem. Phys. 107(14), 5319-5331 (1997).

18. J.-H. Son, "Principle and applications of terahertz molecular imaging," Nanotechnology 24(21), 214001 (2013).

19. K. J. Siebert et al., "All-optoelectronic continuous wave THz imaging for biomedical applications," Phys. Med. Biol. 47(21), 3743-3748 (2002).

20. C. S. Joseph et al., "Continuous wave terahertz transmission imaging of nonmelanoma skin cancers," Laser. Surg. Med. 43(6), 457-462 (2011).

21. P. Doradla et al., "Detection of colon cancer by continuous-wave terahertz polarization imaging technique," J. Biomed. Opt. 18(9), 090504 (2013)

22. H. Chen et al., "Diagnose human colonic tissues by terahertz near-field imaging," J. Biomed. Opt. 20(3), 036017 (2015).

23. H. Liu et al., "Size-controlled synthesis of dendrimer-stabilized silver nanoparticles for X-ray computed tomography imaging applications," Polym. Chem. 1(10), 1677-1683 (2010)

24. R. X. Wang et al., "Microplasma-assisted synthesis of colloidal gold nanoparticles and their use in the detection of cardiac troponin I (cTn-I)," Plasma Processes Polym. 12(4), 380-391 (2015).

25. R. X. Wang et al., "Microplasma-assisted growth of colloidal silver nanoparticles for enhanced antibacterial activity," Plasma Processes Polym. 11(1), 44-51 (2014).

26. A. J. Mieszawska et al., "Multifunctional gold nanoparticles for diagnosis and therapy of disease," Mol. Pharmaceutics 10(3), 831-847 (2013).

Biographies for the authors are not available. 